\title{
Građa
}

\author{
Milan Vrbanus \\ (Hrvatski institut za povijest - Podružnica za povijest \\ Slavonije, Srijema i Baranje, Slavonski Brod)
}

\section{POPIS KRIZMANIKA ŽUPE NAŠICE IZ 1777. GODINE}

UDK 929:265(497.5 Našice) “1777““

Stručni rad

Primljeno: 26. 5. 2020.

U knjižnici Samostana sv. Antuna Padovanskog u Našicama čuva se popis krizmanika našičke župe iz 1777. godine. Popis krizmanika uvezan je, vjerojatno u vrijeme uređenja knjižnice 1987., u Matičnu knjigu vjenčanih župe Našice (1717.-1787.). Matična knjiga čuva se u trezoru samostanske knjižnice među Zapisnicima naredbi provincijala Franjevačke provincije sv. Ivana Kapistrana. ${ }^{1}$

Popis krizmanika sastoji se od 14 rukom pisanih stranica (od 3. do 16. stranice). ${ }^{2} \mathrm{U}$ popisu nedostaju prve dvije stranice, koje očito nisu ostale sačuvane. Naime, prema zapisu fra Silvestra Hungara ${ }^{3}$ u crkvi sv. Antuna Pado-

${ }^{1}$ U Arhivu Samostana sv. Antuna Padovanskog u Našicama čuvaju se originalne korice tog popisa. (Arhiv Franjevačkog samostana sv. Antuna Padovanskog u Našicama, Matična knjiga vjenčanih župe Našice od 1717. do 1787.) - Sign. B - II - 1.

${ }^{2} \mathrm{~S}$ obzirom na numeraciju popisa može se zaključiti da u popisu nedostaju prve dvije stranice. (AFSN, MKV I (1717.-1787.), 3-16)

${ }^{3}$ Silvester Hungar (* Brod, 27. XI. 1745. - † Cernik, 5. I. 1789.), franjevački profesor filozofije i teologije. Pristupio je 8. rujna 1761. u samostanu u Šarengradu u Franjevački red Provincije sv. Ivana Kapistrana, a zavjete je položio u istom samostanu 8. rujna sljedeće godine. Boravio je 1762., kao zavjetovani klerik, u samostanu u Našicama. Filozofiju je studirao od 1764 do 1767. u Osijeku, tijekom čega je 1767. sudjelovao u javnoj raspravi iz filozofije. Ispit za profesora filozofije položio je 1772. u Somboru. Predavao je od 1776. do 1778. na učilištu moralnog bogoslovlja u Našicama, a od 1778. do 1779. obavljao je službu vojnog kapelana u Gradiškoj pukovniji. Sudjelovao je 26. lipnja 1780. u javnoj raspravi iz logike i čiste matema- 
vanskog u Našicama sakrament potvrde primilo je više krizmanika nego što je navedeno u samom popisu. Osim numeracije koju je stavio popisivač, u popisu je stavljena, najvjerojatnije od osobe koja sređivala samostansku knjižnicu, i numeracija od 497. do 509. Pri tome je numeraciju stavio od zadnje prema prvoj stranici, ali prva stranica popisa nije numerirana. ${ }^{4}$

Imena krizmanika navedena su u tri podjednaka stupca. Popisivač je na kraju svake stranice naveo broj krizmanika, a na kraju popisa i ukupni broj krizmanih osoba. Međutim, stvaratelj popisa naveo je na kraju dokumenta broj povrđenih osoba koji je netočan. Naime, netočno je zbrojio broj krizmanih, pa je prema popisivaču zagrebački biskup Josip Galjuf ${ }^{5}$ krizmao 1.565

tike oca Cecilijana Ockla na učilištu u samostanu u Našicama. Službu župnika i gvardijana samostana u Našicama obnašao je od 1780. do 1781., a od 1782. do 1783. te od 1788. do 1789. u samostanu u Cerniku. Tijekom boravka u Cerniku bio je 1782. ispitivač na studiju moralne teologije. Kao cernički gvardijan dobio je 1788. od vojnokrajiških vlasti 150 forinti za obnovu samostana. Služeći bolesne vojnike u cerničkom samostanu, zarazio se i umro. Govorio je hrvatski i talijanski jezik. Franjo Emanuel Hoško, «Dvije osječke visoke škole u 18. stoljeću (II. dio)», Kačić - Zbornik Franjevačke provincije Presvetog Otkupitelja vol. X, 151 bilj. 253, 156; Franjo Emanuel Hoško, Franjevci i poslanje Crkve u kontinentalnoj Hrvatskoj (Zagreb, 2001), 200; Franjo Emanuel Hoško, Franjevačke visoke škole u kontinentalnoj Hrvatskoj (Zagreb, 2002), 274, 282 bilj. 253, 288-289; Franjo Emanuel Hoško, Josip Pavišević - svjedok jozefinizma u Slavoniji i Podunavlju (Zagreb, 2003), 192 bilj. 292, 232, 236; Julije Jančula, Franjevci u Cerniku (Slavonska Požega, 1980), 170, 173-175, 186, 207; Šime Demo, Maja Rupnik-Matasović, Tamara Tvrtković i Milan Vrbanus, Zapisnik franjevačkog samostana u Našicama knjiga I (1739.-1787.) (Našice - Slavonski Brod - Zagreb, 2010), 43, 77, 81, 87, 93, 95, 97, 101, 260; AFSN, Nekrologij, 1998., 7; AFSB, Matica, [22]; http://www.archivum. ferencesek.hu/letoltes/irodalom/Kaizer_Cathalogus.patrum.pdf (14. 06. 2013.)

${ }^{4}$ AFSN, MKV I (1717.-1787.), 3-16, odnosno 497-509.

${ }^{5}$ Josip Galjuf(* Varaždin, 20. XI. 1722. - † Zagreb, 5. III. 1786.), zagrebački biskup od 1772. do 1786. godine. Školovanje je započeo 1738. u Beču. Bogoslovlje je studirao od 1740. do 1744. na kolegiju u Rimu, gdje je 1744. doktorirao. Zaređen je 1744. za svećenika te je 1744. postao župnik u Dubravi. Položio je 9. rujna 1744. vjeroispovijed i župničku prisegu zagrebačkom biskupu Jurju Branjugu za župnika župe Dubrava. Imenovan je 1745. zagrebačkim kanonikom, a sljedeće godine rektorom Hrvatskog kolegija u Bologni. Biskup Klobušický imenovao ga je članom konzistorijalnog vijeća. Postao je 1751. vaškanskim, 1755. kalničkim te 1764. goričkim i bekšinskim arhiđakonom. Kanonik lektor postao je 1763., a 1771. zagrebački prepošt. Zagrebačkim biskupom imenovan je 1772., ali papa ga je potvrdio tek 1777. Prilikom razgraničenja Zagrebačke i Bosansko-srijemske biskupije povjerenstvo je predložilo da se od slavonskog dijela Zagrebačke biskupije osnuje Požeška biskupija, no tome se usprotivio bosansko-srijemski biskup Matija Franjo Krtica te Galjufov izaslanik Antun Mandić. Bio je 1771. prepošt sv. Benedikta de Kaposffe. Tijekom biskupovanja Galjuf je bio kritičan prema jozefinskim uredbama (naročito prema odredbi o ženidbenim zaprekama), slobodoumnim idejama te snošljivom ponašanju prema nekatolicima. Bio je zagovornik hodočašća i proštenja. Zauzimao se za izgradnju bolnice u Zagrebu te je dao izgraditi dom za nemoćne svećenike. Postao je 1757. prisjednik i prelat Banskog stola. Imenovan je kasnije kraljevskim savjetnikom i povjerenikom. (ZC, 1755., [2]; Calendarium Zagrabiense ad Annum Jesu Christi MDCCLX. ..., Zagreb, 1760., [1]; Zagrabiense Calendarium ad annum Jesu Christi MDCCLXXI. ..., 1771., [1]; Zagrabiense Calendarium ad annum Jesu Christi MDCCLXXXI- 
krizmanika, što je netočno jer su sakrament potvrde primile 1.562 osobe, 879 osoba muškog i 683 osobe ženskog spola. ${ }^{6}$

Podjelu sakramenta potvrde zagrebačkog biskupa Josipa Galjufa iz 1777. godine opisao je u Zapisniku franjevačkog samostana sv. Antuna Padovanskoga u Našicama otac Silvester Hungar. Biskup Josip Galjuf stigao je u našički samostan 27. lipnja u pratnji titularnog biskupa Sofije, zagrebačkog kanonika, prepošta čazmanskog kaptola, arhiđakona arhiđakonata Gušće i Vaške Josipa baruna Wernecka, ${ }^{7}$ pobočnog kanonika Antuna Mandića, ${ }^{8}$ budućeg bosansko-

II. ..., Zagreb, 1783., [1]; Zagrabiense Calendarium ad annum Jesu Christi MDCCLXXXV. ..., Zagreb, 1785., [1]; Zagrabiense Calendarium ad annum Jesu Christi MDCCLXXXVI. ..., Zagreb, 1786., [1]; Hoško, Josip Pavišević, 51 bilj. 139; Hrvatski biografski leksikon, 4, 1998., 566-567; Juraj Batelja, Lelja Dobronić, Juraj Kolarić i dr., Zagrebački biskupi i nadbiskupi, Zagreb, 1995., 419-424; Andrija Lukinović, Zagreb - devetstoljetna biskupija, Zagreb, 1995., 259-263; Stjepan Razum, Vjeroispovijedi i župničke prisege Zagrebačke nadbiskupije - Professiones fidei et iuramenta parochorum ecclesiae Zagrabiensis 1648.-1997., Zagreb 2010., 168)

${ }^{6}$ AFSN, MKV I (1717.-1787.), 3-16, odnosno 497-509.

${ }^{7}$ Josip Werneck (* Brinje, 1729. - † Zagreb, 16. IV. 1779.), svećenik Zagrebačke biskupije. Teologiju je studirao u Rimu, gdje je do 1752. bio pitomac Njemačkog zavoda sv. Apolinara kao svećenik Senjsko-modruške biskupije, a nakon toga Zagrebačke biskupije. Zagrebački biskup Franjo Thauszy primio ga je usprkos protivljenju zagrebačkih kanonika 1752. među svećenike Zagrebačke biskupije. Od 1755. do 1765. obnašao je službu župnika u Lipiku. Položio je u travnju 1755. vjeroispovijed i župničku prisegu zagrebačkom biskupu Franji Thauszyju za župnika župe u Lipniku. Župnik u Požegi postao je 1764. Zagrebačkim kanonikom imenovan je 1765., a 1769. čazmanskim prepoštom. Bio je 1771. prefekt sjemeništa Zagrebačke biskupije. Od 1769. do 1777. obavljao je kanonske vizitacije arhiđakonata Gušće i Since. Bio je 1776. savjetnik Hrvatskog kraljevskog vijeća te naslovni biskup Sofije. Zagrabiense Calendarium ad annum Jesu Christi MDCCLXXI. ..., 1771., [1]; Rudolf Horvat, «Slavonski Brod», Hrvatska prošlost knj. I. (Zagreb 1940), 113; Hoško, Josip Pavišević, 23 bilj. 26; Razum, Vjeroispovijedi, 177.

${ }^{8}$ Antun Mandić (* Požega, 16. VIII. 1740. - † Đakovo, 11. I. 1815.), bosansko-đakovački i srijemski biskup od 1806. do 1815. Nižu i srednju školu polazio je u Požegi. U sjemenište je primljen u Zagrebu, gdje je završio retoriku. Prisegnuo je 1757. na Bečkom kolegiju, gdje je završio filozofiju. Teologiju je počeo studirati 1759. u Bologni, ali je studij zbog bolesti završio u Beču. Za svećenika je zaređen 1763. Od 1763. do 1765. vršio je službu rektora sjemeništa u Požegi. Od 1765. do 1769. upravljao je župom u Slavonskom Kobašu, a od 1769. do 1771. bio je župnik iste župe, dok je od 1771. do 1779. bio župnik župe Lipovljani. Položio je 11. kolovoza 1769. vjeroispovijed te župničku prisegu zagrebačkom biskupu Franji Thauszyju za župnika župe Kobaš, a 20. travnja 1771. za župnika župe Lipovljani. Postao je 1775. začasnim kanonikom čazmanskog kaptola, kanonikom biskupa Galjufa i vicerektorom Hrvatskog kolegija u Beču na preporuku arhiđakona Wernecka. Imenovan je nadzornikom škola za Hrvatsku i Slavoniju 1776., te savjetnikom dvorskog savjetnika baruna Józsefa Ürményija, zaduženoga za uređenje školstva u Ugarskoj i pridruženim kraljevinama. Kao nadzornik škola proveo je široku djelatnost na razvoju školstva u Hrvatskoj i Slavoniji. Teologiju je doktorirao 1777. na Teološkom fakultetu u Beču. Sljedeće godine postao je kanonik zagrebačkog Stolnog kaptola, a 1780. opat Blažene Djevice Marije od Petrovaradina. Bio je osobni delegat zagrebačkog biskupa Josipa Galjufa u komisiji za razgraničenje biskupija u Slavoniji 1781. Iste ga je godine Josip II. imenovao predsjednikom komisije za redakciju 
đakovačkog i srijemskoga biskupa, čazmanskog kanonika Ivana Jambrekovića $^{9}$ te čazmanskog kanonika i ceremonijara biskupa Zagrebačke biskupije

Stullijevog rječnika, a 1782. savjetnikom Ugarskog namjesničkog vijeća. Biskup Galjuf imenovao ga je 1786. osobnim generalnim vikarom. Postao je naslovni prištinski biskup 1788 ., kustod Stolnog kaptola 1792., a 1800. opat Desnice sv. Stjepana, zagrebački prepošt i vranski prior. Proveo je odluku Ugarskog namjesničkog vijeća iz 1789. o podjeli župe Našice na više župa. Od 1790. do 1806. obavljao je dužnost savjetnika Ugarskog namjesničkog vijeća. Franjo I. imenovao ga je 17. lipnja 1806. bosansko-srijemskim biskupom, što je Sveta Stolica potvrdila 26. kolovoza, pa je 18. rujna bio posvećen za biskupa. Osnovao je sjemenište Bosansko-srijemske biskupije 1806. u Đakovu. Tijekom biskupovanja radio je na obnovi biskupije te razvoju đakovačkog vlastelinstva. Bio je tajni savjetnik carsko-kraljevskog veličanstva te nositelj Reda sv. Stjepana. Marin Srakić, «Antun Mandić, biskup, realizator školskih reforma u Hrvatskoj», u: Zbornik o Marijanu Lanosoviću (Osijek, 1985), 87-97; Franjo Emanuel Hoško, Franjevci u kontinentalnoj Hrvatskoj kroz stoljeća (Zagreb, 2000), 245-246; Emerik Gašić, Kratki povijesni pregled biskupija Bosansko-đakovačke i Srijemske (Osijek, 2000), 74, 126, 150; Julije Kempf, Požega - zemljopisne bilješke iz okoline i prilozi za povijest Slob. $i$ kr. grada Požege i Požeške županije, pretisak iz 1910. (Jastrebarsko, 1995), 602-603; Hoško, Poslanje Crkve, 179 bilj. 16; Hoško, Josip Pavišević, 47, 117 bilj. 55; Razum, Vjeroispovijedi, 195-196; www.literature.at/viewer.alo?objid $=11769 \&$ viewmode $=$ fullscreen\&scale $=2 \&$ page $=370$ (22. 01.2010.)

${ }^{9}$ Ivan Jambreković (* ? - † Vrapče, 27. III. 1821.), župnik u Vrapču. Bio je 1760. kanonik Čazmanskog kaptola i prebendar Zagrebačke biskupije. Obavljao je 1777. službu kanonika Čazmanskog kaptola. Bio je 1777. u pratnji zagrebačkog biskupa Josipa Galjufa u vizitaciji našičke župe. Obavljao je 1787. službu župnika župe u Vrapču. Položio je 4. travnja 1792. vjeroispovijed i župničku prisegu zagrebačkom biskupu Maksimilijanu Vrhovcu za župnika župe u Vrapču. Službu župnika župe u Vrapču obavljao je od 1792. do svoje smrti. Calendarium Zagrabiense ad Annum Jesu Christi MDCCLX. ..., Zagreb, 1760., [2]; Zagrabiense Calendarium ad annum Jesu Christi MDCCLXXXVII. ..., 1787., [7]; Zagrabiense Calendarium ad annum Jesu Christi MDCCXCIII. ..., Zagreb, 1793., [5]; Zagrabiense Calendarium ad annum Jesu Christi MDCCXCIV. ..., Zagreb, 1794., [5]; Zagrabiense Calendarium ad annum Jesu Christi MDCCXCV. ..., Zagreb, 1795., [5]; Zagrabiense Calendarium ad annum Jesu Christi MDCCXCVI. ..., Zagreb, 1796., [5]; Zagrabiense Calendarium ad annum Jesu Christi MDCCXCVII. ..., Zagreb, 1797., [7]; Zagrabiense Calendarium ad annum Jesu Christi MDCCXCVIII. ..., Zagreb, 1798., [7]; Zagrabiense Calendarium ad annum Jesu Christi MDCCXCIX. ..., Zagreb, 1799., [5]; Zagrabiense Calendarium ad annum Jesu Christi MDCCC. ..., 1800., Zagreb, 1800., [8]; Zagrabiense Calendarium ad annum Jesu Christi MDCCCI. ..., 1801., Zagreb, 1801., [5]; Zagrabiense Calendarium ad annum Jesu Christi MDCCCII. ..., Zagreb, 1802., [7]; Zagrabiense Calendarium ad annum Jesu Christi MDCCCIII. ..., Zagreb, 1803., [7]; Zagrabiense Calendarium ad annum Jesu Christi MDCCCIV. ..., Zagreb, 1804., [7]; Zagrabiense Calendarium ad annum Jesu Christi MDCCCV. ..., Zagreb, 1805., [5]; Zagrabiense Calendarium ad annum Jesu Christi MDCCCVI. ..., Zagreb, 1806. , [8]; Zagrabiense Calendarium ad annum Jesu Christi MDCCCVII. ..., Zagreb, 1807., [9]; Zagrabiense Calendarium ad annum Jesu Christi MDCCCVIII. ..., Zagreb, 1808., [5]; Zagrabiense Calendarium ad annum communem Jesu Christi MDCCCXI. ..., Zagreb, 1811., 46; Zagrabiense Calendarium ad annum communem Jesu Christi MDCCCXV. ..., Zagreb, 1815. 53; Zagrabiense Calendarium ad annum communem Jesu Christi MDCCCXVIII. ..., Zagreb, 1818., 53; Zagrabiense Calendarium ad annum bissextilem Jesu Christi MDCCCXX. ..., Zagreb, 1820., 57; Zagrabiense Calendarium ad annum communem Jesu Christi MDCCCXXI. ..., Zagreb, 1821., 57; Demo, Rupnik-Matasović, Tvrtković i Vrbanus, Zapisnik, 79; Razum, 
Petra Rebrovića. ${ }^{10}$ Biskup je podjelu sakramenta potvrde obavljao tri dana (od 27. do 29. lipnja). Prvog dana sakrament potvrde primilo je 1.312 krizmanika, drugog 389, a trećeg dana fra Silvester Hungar nije zabilježio u Zapisniku broj osoba koje su primile sakrament potvrde. ${ }^{11}$

Iz Hungarovog zapisa može se zaključiti da je zagrebački biskup Josip Galjuf u tri dana krizmao više krizmanika nego što je to popisao sastavljač popisa krizmanika. Nažalost, nije moguće utvrditi točan broj krizmanika, ali se može s velikom sigurnošću pretpostaviti da ih je bilo više nego što ih je zabilježeno u popisu. Naime, prema podacima u Zapisniku može se zaključiti da je u prva dva dana potvrdu primila 1.701 osoba, što je više nego što je navedeno u popisu. S obzirom na to da je biskup Galjuf sakramenat potvrde dijelio i treći dan određenom broju osoba, može se pretpostaviti da je na kraju sigurno krizmano više osoba nego što je navedeno na kraju popisa. ${ }^{12}$

Potvrdu moje pretpostavke moguće je naći i u nepotpunom ${ }^{13}$ sumarnom status animarum po naseljima župe Našice iz 1771. s imenima nositelja kućanstva, sumarnom status animarum iz iste godine te sumarnom popisu katoličkog stanovništva iz 1775. godine. Prema sumarnom status animarum iz

Vjeroispovijedi, 217, 266; Nadbiskupijski i kaptolski arhiv Zagrebačke nadbiskupije, Matica umrlih svećenika, 46.

${ }^{10}$ Petar Rebrović (* ? - † Nart, 31. I. 1793.), svećanik Zagrebačke biskupije. Obavljao je 1777. službu kantora i čazmanskog kanonika te ceremonijara zagrebačkog biskupa Josipa Galjufa. Bio je 1777. u pratnji zagrebačkog biskupa Josipa Galjufa u vizitaciji našičke župe. Vršio je od 1778. do 1786. službu kanonika Čazmanskog kaptola i prebendara. Položio je 1785. vjeroispovijed i župničku prisegu zagrebačkom biskupu Josipu Galjufu za župnika župe u Nartu, što je ostao do svoje smrti. Obavljao je od 1789. do smrti službu vicearhiđakona okruga Nart. Zagrabiense Calendarium ad annum Jesu Christi MDCCLXXXIII. ..., Zagreb, 1783., [3]; Zagrabiense Calendarium ad annum Jesu Christi MDCCLXXXV. ..., Zagreb, 1785., [23]; Zagrabiense Calendarium ad annum Jesu Christi MDCCLXXXVI. ..., Zagreb, 1786., [3, 5-6]; Zagrabiense Calendarium ad annum Jesu Christi MDCCLXXXIX. ..., Zagreb, 1789., [2, 4]; Zagrabiense Calendarium ad annum Jesu Christi MDCCXCI. ..., Zagreb, 1791., [2]; Zagrabiense Calendarium ad annum Jesu Christi MDCCXCII. ..., Zagreb, 1792., [2, 5]; Zagrabiense Calendarium ad annum Jesu Christi MDCCXCIII. ..., Zagreb, 1793., [2, 5]; Régi és Új Kalendariom, mellt Kristus Urunk Szúletése Után-Való MDCCLXXVIII. ..., 1778., 78; Régi és Új Kalendariom, mellt Kristus Urunk Szúletése Után-Való MDCCLXXXII. ..., 1782. 84-85; Vetus et Novum Calendarium ad annum a nativitate Salvatoris nostri Jesu Christi MDCCLXXXX. ..., 1790., 77; Demo, Rupnik-Matasović, Tvrtković i Vrbanus, Zapisnik, 79; Razum, Vjeroispovijedi, 209; NAZ, MUS, 8.

${ }^{11}$ AFSN, Protocollum, Tom. I, fol. 45-46; Demo, Rupnik-Matasović, Tvrtković i Vrbanus, Zapisnik, 77, 79.

${ }^{12}$ AFSN, Protocollum, Tom. I, fol. 45-46; Demo, Rupnik-Matasović, Tvrtković i Vrbanus, Zapisnik, 77, 79.

${ }^{13}$ U sumarnom poimeničnom status animarum župe Našice iz 1771. nedostaju poimenični popisi sela Zoljan i Seona. U sumarnom status animarum iz iste godine navedeni su sumarni podaci za spomenuta naselja. AFSN, bez bliže arhivske oznake. 
1771. u župi Našice bilo je tada krizmano 2.161 osoba muškog i ženskog spola od ukupno 4.067 katolika, što znači da je bez sakramenta potvrde bilo 1.906 osoba ili 46,87 \% svih katolika. Prema status animarum iz 1775. u župi Našice pripravnih osoba za sakrament potvrde bilo je 1.814 ili 44,33 \% od ukupnog broja pripadnika katoličke vjere na području župe. ${ }^{14}$

Kanonska vizitacija, koju je 16. svibnja 1783. obavio vaškanski arhiđakon Josip Taisperger, donosi i status animarum župe Našice, u kojem su prikazani i podaci o broju krizmanih osoba. Prema status animarum iz 1783. na području župe Našice bilo je krizmano 3.045 osoba ili 64,46 \% svih katolika, što znači da je u župi bez sakramenta potvrde bilo 1.679 ili 35,54 \% stanovnika katoličke vjere u župi Našice. ${ }^{15}$

Za razliku od župe Našice, u župama valpovačkog vlastelinstva (Valpovo, Marijanci, Šljivoševci, Koška, Miholjac, Petrijevci i Brođanci) ${ }^{16}$ udio osoba pripravnih za primanje sakramenta potvrde bio je 1767. znatno niži od udjela takvih osoba u župi u Našicama. Naime, u župama valpovačkog vlastelinstva njihov se udio kretao između nešto više od $1 /$ svih katolika $(14,82 \%)$ u župi Miholjac do nešto manje od $3 / 7$ svih pripadnika katoličke vjere $(41,60 \%)$ u župi Marijanci. Prema navedenim podacima, udio osoba pripravnih za podjelu ovog sakramenta bio je najbliži župi Našice u župi Marijanci. U ostalim župama valpovačkog vlastelinstva udio osoba pripravnih za podjelu sakramenta potvrde bio je znatno niži. Naročito to vrijedi za župe Miholjac, Valpovo i Petrijevci, koje su, osim župe Marijanci, bile najveće župe na području vlastelinstva. ${ }^{17}$

Na temelju iznesenih činjenica može se pretpostaviti da je krajem lipnja 1777. godine sakrament potvrde primilo sigurno više osoba nego što je navedeno u popisu krizmanika, ali nije moguće preciznije reći o kojem je broju riječ.

Nažalost, stvaratelj popisa, osim broja imena krizmanika, nije naveo ni jedan drugi podatak o krizmanicima, primjerice imena naselja, životnu dob,

\footnotetext{
${ }^{14}$ AFSN, bez bliže arhivske oznake.

${ }^{15}$ NAZ, Kanonske vizitacije, Prot. knj. 181, fol. 109; AFSN, Župni arhiv, Knjiga vizitacije Puncta visitationis, B- XXIII - 1, spis br. 16.

${ }_{16}$ Župe na valpovačkom vlastelinstva utvrdio sam na temelju status animarum katoličkih župa na valpovačkom vlastelinstvu iz 1754. Prema tom dokumentu na vlastelinstvu su tada postojala navedene župe. Prema uvodnoj studiji priređivača Popisa katoličkog stanovništva u istočnoj Slavoniji 1767. godine na valpovačkom vlastelinstvu postojale su župe Petrijevci, Valpovo, Donji Miholjac i Koška. Državni arhiv u Osijeku, HR-476, Valpovačko vlastelinstvo, Acta Viennensia, kut. br. 23, spis br. 571; Denis Njari, Popis katoličkog stanovništva u istočnoj Slavoniji 1767. godine - A kelet-szlavóniai katolikus lakosság 1767. évi összeírása (Osijek, 2019), 34 i 65.
}

${ }^{17}$ Njari, Popis katoličkog stanovništva, 369-678. 
bračno stanje itd., ${ }^{18}$ koji bi istraživačima omogućili analizu broja krizmanika prema naseljima, dobnu strukturu i njihovo bračno stanje prema spolu te druge demografske karakteristike stanovništva našičke župe sv. Antuna Padovanskog. Također, takvi bi podaci omogućili utvrđivanje demografskih karakteristika krizmanika. Primjerice, bilo bi zanimljivo utvrditi prosječnu dob krizmanika jer na današnjem stupnju istraženosti nemoguće je tvrditi u kojoj su se dobi krizmale osobe u Slavoniji, ali i Hrvatskoj. Navedeni podatak bio bi zanimljiv s obzirom na činjenicu da u vrijeme nastanka popisa sakrament potvrde nije podjeljivan svake godine kao danas, već samo kada je biskup bio prisutan u župi, pa je dob krizmanika bila puno veća nego što je to danas slučaj.

\section{KORIŠTENE KRATICE}

] razdvaja natuknicu od ostatka kritičkog aparata

(!) upravo tako napisano

(?) upitna točnost transkripcije

$[\ldots]$ dopunjeni dio riječi

$\{\ldots\}$ nedostaje dio teksta

cancell. (cancellavit) precrtao

corr. (correxit) ispravio

desunt nedostaju

pag. (pagina, paginae) stranica, stranice

${ }^{18}$ AFSN, MKV I (1717.-1787.), 3-16. 
$\{\ldots\}^{19}$

\section{/p. 3/ Catalogus confirmatorum per excellentissimum ac reverendissimum dominum Iosephum Galyuff episcopum Zagrabiensem 1777. in ecclesia sancti Antonii Patavinni Nashicis}

Anna Vinkovich
Eva Szidarich
Agata Vidakovich
Francisca Johancsevich
Maria Staglich
Anna Micsich
Helena Ivancsich
Anna Benovich
Anna Volarevich
Hena Maksan
Rosa Boxich
Catharina Berkich
Rosa Jednacsich
Lucia Peich
Theresia Ridlin
Margaretha Balaczkovich
Jacobus Zomborlich
Helena Remich
Hena Pavlovich

Heva Littarich

Johannes Stuparich

Antonius Batrakovich

Blasius Shibalich

Johannes Kovacsich

Andreas Priticsich

Thomas Priticsich

Marcus Mihakovich
Anna Amicsich

Francisca Tupovich

Georgius Lovrich

Barbara Blaxanovich

Josephus Tüpelhoffer

Michael Berlokovich

Georgius Medich

Heva Berkich

Anna Drucsalovich

Petrus Shepcsich

Catharina Sjuralich

Anna Sjuralich

Franciscus Bartholovich

Catharina Shimich

Heva Shimich

Margaretha Littarich

Margaretha Shivadinovich

Magdalena Bartholovich

Juliana Shimich

Antonius Siecsan

Johannes Siecsan

Michael Csumlich

64

/p. 4/

Nicolaus Bolkovacz

Matthaeus Bolkovacz

Johannes Mihelcsich

Antonius Stevich

Stanislaus Dumich

Rosa Stojicsevich

Rosa Glavashevich

Maria Ivanovich
Nicolaus Knexevich

Mathias Knexevich

Ladislaus Shokicsich

Martha Novakovich

Brigitta Filipovich

Anastasia Bosnjakovich

Anna Blaxevich

Anna Kapetanovich

Barbara Dumich

Bartholomaeus Blaxevich

Stanislaus Pritixanac

Catharina Matokovich

Catharina Sharcsevich

Johannes Vukomanovich

Magdalena Benich

Coleta Benich

Lucas Tomashevich

Heva Sharich

Johanna Sharich

Georgius Petrich

Paullus Pavich

Heva Marinovich

Magdalena Dolaczkovich

Marcus Littarich

Georgius Littarich

Lucia Benakovich

Maria Kovacsevich

Marian Belobradich

Johannes Blagalacz

Johannes Kverxich

Magdalena Csakalich

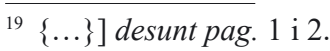


Catharina Zdelarovich

Antonia Babich

Jacobus Matokovich

Marcus Blaxevich

Catharina Vinkovich

Juliana Blaxicsich

Anna Kolarich

Bartholomaeus Pauer

Simon Shimanovich

Joseph Shimanovich

Anna Vidakovich

Catharina Minorich

Angela Dorich

Rosalia Ivanovich

Andreas Knexevich

Antonius Stoxich

Michael Stoxich

Josephus Littarich

Antonius Garaga

Thomas Littarich

Antonius Pritishanac

Paullus Littarich

Thomas Bartholovich

Georgius Roskovich

Jacobus Bilokapich

33

Jacobus Kurtak

Catharina Mihalenovich

Magdalena Mathianich

Helena Thomelich

Nicolaus Derdanovich

Antonius Majstorovich

Adamus Poljak

Marcus Ugarkovich

Adamus Mikicsich

Angela Anicsich

Dorothea Balatovich

Heva Balatovich
Lucas Kulanzovich

Marcus Balatovich

Elias Ocselich

Rosalia Markovich

Maria Mihich

Birgita Gjurich

Paullus Biuklich

Johanna Prisbilovich

Magdalena Udovicsich

Adam Bartholovich

Johanna Ivanovich

Maria Grussich

Lucas $^{20}$ Vukshich

Benedict Vukshich

Michael Garacsich

Emericus Kovacsich

Michael Druczalovich

Adam Xivkovich

Dorothea Stanislavich

Magdalena Stanislavich

Anna Horvatovich

Maria Druczalovich

Catharina Druczalovich

Paullus Pritishanacz

Nicolaus Berdarich /p. $5 /$

Michael Markovich

Catharina Hebicsich

Raphael Shivatinovich

Lucia Mathokovich

Elias Jelich

Agata Blaxevich

Rosa Darjanacz

Anna Micsich

Theresia Littarich

Michael Anicsich

Thaddaeus Kovacsevich

Petrus Thomashevich
Maria Zidar

Coecilia Pishich

Coleta Pishich

Catharina Divianovich

Magdalena Poljak

Catharina Blaxevich

Stanislaus Rascsanacz

Georgius Mitrakovich

Franciscus Cvitovich

Michael Matishich

Nicolaus Filipovich

Josephus Kapralis

Paullus Pavishich

Lucia Benovich

Andreas Matishich

Lucas Zvonarovich

Thaddaeus Zvonarovich

Bartholomaeus Berkich

Marianus Shimich

Michael Sharcsevich

Petrus Paljovich

Marianus Stuparich

Andreas Bortich

Antonius Damnjanovich

Paullus Szidar

Elias Kurtak

Martinus Kurtak

35

Georgius Petrovich

Adamus Jacoalacz

Marianus Mathishich

Mathias Littarich

Elias Littarich

Thomas Littarich

Antonius Matokovich

Mathias Sdelich

Magdalena Ocselich

Martinus Klobucsar

Johanna Cvitovich

Catharina Pavlovich

20 Lucas] Luas 


Thomas Pritishanacz
Lucia Katavich
Rosa Tupovich
Johannes Minovich
Johannes Remetich
Heva Lovashich
Anna Puanich
Francisca Shimich
Maria Littarich
Anna Krocerinn
Maria Stojicsich
Josephus Vucsenovich
Heva Littarich
Theresia Balaczkovich
Laurentius Spoljarovich
Michael Vucsinovich
Magdalena Littarich
Margareta Lukshich
Georgius Mihaljevich
Antonius Vucsinovich
Jacobus Berich
Catharina Matokovich
Paullus Biuklich
Antonius Vucsinovich
Johannes Berich
Heva Damnjanovich
38
38

Marcus Stucsich

Helena Kuzmanovich

Elisabetha Habicsich

Maria Habicsich

Elisabetha Matokovich

Magdalena Kovacsevich

Anna Ancsich

Barbara Ivancsich

Agatha Benovich

Josephus Thomashevich

Nicolaus Sharich

Anna Millinovich

Catharina Pavull

Magdalena Shimovich

Antonius Paljugovich
Dominicus Kovacsich

Stephanus Glasovacz

Antonius Jovich

Petrus Nikolich

Clara Shimich

Maria Jakocsevich

Heva Pajakovich

Rosa Slopetich

Lucia Udovesich

Rosa Prispilovich

Georgia Blaxevich

Catharina Jugovich

Coleta Matokovich

Monica Matokovich

Rosa Matishich

Catarina Matokovich

Monica Matokovich

Rosa Matokovich

Rosa Cvitich

Catharina Sharich

Maria Sharich

Apollonia Damnjanovich

Lucia Radacsich

Francisca Divianovich

Rosa Shimunovich

37

/p. 6/

Antonius Rupcsich

Maria Knexevich

Maria Zalovich

Catharina Vukoshich

Johannes Kovacsich

Bartholomaeus Blaxevich

Franciscus Biuklich

Paullus Blaxavich

Antonius Miocsevich

Marcus Biuklich

Emericus Marich

Helena Shubich

Johannes Gherbeshich

Marcus Bolikovich

Marcus Boshnjak
Magdalena Pavlovich

Mathias Mihokovich

Georgius Bartholovich

Anastasia Stojicsich

Petrus Szigura

Antonius Berlonakovich

Petrus Juxevich

Philippus Jankovich

Georgius Glasovacz

Matthaeus Glasovacz

Josephus Cernkovich

Lucas Cernkovich

Marcus Draghich

Matthaeus Kovacsevich

Stephanus Glavashevich

Barbara Karoly

Elisabet Kramareck

Heva Debeljakovich

Anna Matkovich

Agatha Ghemovich

Heva Starcsevich

Klara Kossovich

Rosa Blaxanovich

Augustinus Gjukanovich

Adamus Likarich

\section{7}

Michael Knexevich

Matthaeus Ugarkovich

Elias Matarich

Joannes Gjurichich

Michael Kovacsevich

Franciscus Blaxevich

Marianus Klaich

Marcus Vidacsich

Marian Vidacsich

Marianus Biuklich

Antonius Biuklich

Anna Lovrich

Agnes Lovrich

Marcus Blaxevich

Josephus Thadiich (!) 


Maria Marovich
Matthaeus Schneperger
Mathias Blaxicsevich
Petrus Mrakovich
Matthaeus Johovich
Josephus Erich
Georgius Jazvanacz
Antonius Frankovich
Jacobus Benovich
Johannes Shimich
Nicolaus Golubovich
Antonius Pavoshevich
Matthaeus Ghergich
Rosa Kovacsevich
Nicolaus Klobucsar
Rosa Frankovich
Johanna Humilianovich
Catharina Kovacsevich
Antonius Habicsich
Thomas Kukolovich
Petrus Bencsevich
Johannes Kovacsevich
Carolus Cernovich
Petrus Udovicsich

39

Jacobus Horvatovich

Antonius Kranich

Marcus Krunich

Johannes Stojicsich

Antonius Stojicsich

Paullus Stojicsich

Johannes Stojicsich

David Belakovich

Andreas Kapetanovich

Maria Sharcsevich

Cecilia Glasovacz

Maria Glasovacz

Catharina Domazetovich
Lucas Agich

Petrus Abcsich ${ }^{21}$ (!)

Josephus Thomashevich

Stanislaus Knexevich

Georgius Biuklich

Franciscus Babich

Johannes Knexevich

Paullus Kovacsevich

Laurentius Paljushich

Jacobus Mathokovich

Marian Jakobovich

Michael Mostanievich

Heva Novalich

Anna Shimich

Theresia Shimanovich

Heva Krucsevacz

Johannes Orozkovich

Antonius Orozkovich

Marcus Mathishich

Petrus Knexevich

35

/p. $7 /$

Anna Filipovich

Agatha Vidakovich

Magdalena Shustich

Lucas Glasovacz

Maria Tucsevich

Philippus Crushich

Michael Glasovacz

Matthaeus Pidishanacz

Josephus Filipovich

Franciscus Filipovich

Paullus Filipovich

Josephus Prispilovich

Marcus Jakovacz
Josephus Ivancsich

Martinus Ivancsich

Lucas Nikcz

Franciscus Zvekan

Antonius Zvekan

Johannes Prispilovich

Magdalena Remetich

Heva Peroshevich

Emericus Rebics

Andreas Staglich

Bonus Kovacsevich

Bartholomaeus

Kovacsevich

Johannes Kovacsevich

Andreas Vidacsich

Adamus Vidacsich

Petrus Sheovich

Mathias Damnianovich

Magdalena Novoselacz

Heva Anicsich

Antonius Blagoëvich

Johannes Blagoevich

Antonius Blaxevich

37

Agnes Shimicsich

Elisabet Pritishanacz

Margaret Mastanievich

Justina Mastanievich

Maria Blaxich

Anna Blaxich

Maria Erich

Francisca Erich

Catharina Ocelich

Magdalena Filipovich

Maria Palashich (!)

Lucia Paloshich

Gregorius Benakovich

$\overline{{ }^{21} \text { Stvaratelj popisa }}$ zabilježio je prezime kao Abcsich, a vjerojatno bi trebalo Abicsich ili Abacsich. 
Maria Domazetovich Helena Blaxanovich Maria Bosnjakovich Anna Bosnjakovich + Heva Bosnjakovich Josephus Novakovich Marcus Novakovich Petrus Thadiich (!) Josephus Bukshich Stephan Bartolovich Nicolaus Bartolovich Marcus Damnjanovich Jacobus Boshnjakovich Georgius Boshnjakovich Jacobus Peicsevich Francisca Jakobljevich Francisca Gjuranovich Francisca Blaxanovich Juliana Matishkovich Johannes Andrich Laurentius Andrich

Petrus Jakobcsevich

Matthaeus Jakobcsevich

Petrus Jovich

Marian Kovacsevich

Michael Gjurcsich

Stephanus Jossich

Petrus Zutinovich

Matthaeus Umilianovich

Cecilia Zecsevich

Agnes Novakovich

Andreas Filipovich

Johanna Mrakovich

Rosa Mucsich

Anna Lovrich

Georgius Lukacsich

Matthaeus Rushevacz Theresia Thomacz
Josephus Ratkovich

Marcus Mikicsich

Matthaeus Mikicsich

Marianus Gjanich

Adamus Gjanich

Elisabet Miokovich

Lucia Baloshich

Anna Cernkovich

Heva Lovrich

Brigita Lovrich

Heva Lovrich

Antonius Abramovich

Nicolaus Ichovich (?)

Anna Mastanievich

Catharina Mastanievich

Maria Sharich

Agnes Sharich

Rosa Sharich

Stephanus Sharich

Michael Stevich

Michael Fabianovich

Thomas Pavlecsich

Nicolaus Vrenich

Thomas Vrenich

Matthaeus Bartolovich

Antonius Jager

Rosa Pavshich

Magdalena Poljacz

Lucia Berdarich

Mathias Boljkovacz

Marcus Lukacisch

44

/p. 8/

Maria Balatovich

Helena Ocelich

Angela Blaxevich

Anna Lovoshich

Antonius Lucsich

Josephus Lucsich

Lucas Zlopircsich ${ }^{22}$

Magdalena Stefancsich

Magdalena Jurkovich
Bartolomaeus Belajcsich

Emericus Belajcsich

Elias Majurcsevich

Lucas Majurcsevich

Matthaeus Shepcsich

Matthaeus Micsich

Marianus Tupovich

Catharina Biuklich

Anna Lovashich

Thoma Opancsarevich

Josephus Bartolovich

Antonius Thomashevich

Andreas Thomashevich

Clara Thomashevich

Rosa Thomashevich

Magdalena Thomashevich

Johannes Miocsevich

Anna Sarcsevich

Juliana Cernovich

Matthiana Milinkovich

Anna Xivkovich

Anna Jankovich

Brigita Thomich

Josephus Muskon

Josephus Blagoevich

Stephanus Kapetanovich

Antonius Ghergurich

Maria Balatovich

Magdalena Perishich

Philippus Zvonarevich

Joannes Zvonarevich

44

Heva Palushich

Catharina Joshavich

Maria Glasovich

Magdalena Patajacz

Catharina Paljushich

Maria Jazvanacz

Magdalena Pritishanacz

Magdalena Dolakovich

Coleta Lovoshevich

${ }_{22}$ Stvaratelj popisa prekrižio je Lucas Zlopircsich, pa ponovo napisao isto ime. 
Theresia Blagajacz

Maria Ghergich

Antonius Stipanovich

Magdalena Vidacsich

Adamus Dorich

Marianus Joshovacz

Mathias Joshovacz

Catharina Mihelcsich

Angela Boljkovacz

Helena Tandakovich

Maria Balatovich

Anna Vukshich

Maria Glasovacz

Angela Glassovacz

Rosa Mirtich

Rosa Kovacsich

Brigita Pavich

Martinus Kovacsevich

Simon Rokovich

Magdalena Prishanacz (!)

Heva Pritishanacz

Josephus Filipovich

Michael Filipovich

Matthaeus Xivadinovich

Josephus Pritishanacz

Heva Ciprianovich

Maria Rakacsich

Paullus Benich

Mathias Benich

Elias Vidovich

Josephus Vidovich

Georgius Divjanovich

Antonius Divjanovich

42

Maria Kovacsevich

Rosa Kovacsevich

Magdalena Vojnich

Georgia (?) Vojnich

Rosa Gjuranovich

Maria Migich

Matthaeus Littarich

Marcus Littarich
Antonius Vinkovich

Adamus Lovrich

Marcus Andrich

Lucas Kovacsevich

Laurentius Kovacsevich

Anna Trusich

Maria Horvatovich

Catharina Mikinich

Nicolaus Petrovich

Johannes Ocelich

Matthaeus Matishich

Stanislaus Kulauzovich

Elias Vicsich

Nicolaus Zalovich

Heva Studinger

Maria Miokovich

Lucia Blaxicsevich

Lucia Peroshevich

Lucia Crushich

Lucia Ognjenovich

Antonius Divianovich

Georgius Ticsich

Thomas Jednacsich

Adamus Ivanovich

Stephanus Petrovich

Simon Marinovich

Heva Dorich

Lucia Prispilovich

Maria Micsich

Agata Shimanovich

Maria Shimanovich

40

/p. 9/

Catharina Littarich

Anna Koprivesich

Magdalena Brodarich

Magdalena Mathishich

Dorothea Gherbacsich

Catharina Ivankovich

Antonius Xivcsich

Andreas Stankovich
Magdalena Barich

Magdalena Vidakovich

Anna Pritishanacz

Catharina Pritishanacz

Agnes Anicsich

Anna Anicsich

Magdalena Perich

Catharina Vinnich

Elisabet Zidarovich

Johannes Pavicsevich

Marcus Antolovich

Thaddaeus Prispilovich

Catharina Remetich

Clara Semialaz

Heva Kovacsevich

Johanna Zidarovich

Apollonia Dashovich

Johannes Mielcsich

Georgius Kvaternik

Blasius Migich

Thomas Migich

Antonius Matokovich

Elisabet Matokovich

Agnes Mihaljevich

Martinus Horvatovich

Matthaeus Bertich

Oliva Matokovich

Marcus Matokovich

37

Hieronymus Majurcsevich

Josephus Vladicsich

Michael Jakshetich

Laurentius Pejcsevich

Francisca Kovacsevich

Magdalena Paraga

Lucia Vdovesich

Magdalena Sharich 


Maria Mathishich
Catharina Vucsinovich
Magdalena Vucsinovich
Antonius Mikich
Lucas Pavoshevich
Thomas Pavoshevich
Antonius Pavoshevich
Nicolaus Lovcsich
Josephus Lovcsich
Josephus Peroshevich
Marianus Petroshich
Dorothea Kovacsich
Heva Kovacsich
Andreas Tominovich
Philippus Sigur
Josephus Shimich
Catharina Kapetanovich
Lucia Rupcsich
Juliana Gjurassovich
Maria Starcsevich
Michael Golich
Bartholomaeus Plivarich
Antonius Plivarich
Marcus Vidakovich
Maria Lucich
Euphemia Ljashetich
David Gregoshevich
Francisca Gherghich
Antonia Starcsevich
Juliana Ljashetich
Catharina Gjurassovich
Rosa Vicich
Helena Mikich
Juliana Franich
Francisca Franich
Heva Zidarovich

Andreas Blaxanovich

Bernardin Gabranovich

Paullus Palushich

Georgius Dorich

Laurentius Dorich

Rosa Zecsevich

Anastasia Filipovich

Angelia Vidacsich

Clara Lukcsich

Anna Bassarovich

Anna Milinkovich

Georgius Mandushich

Johannes Resch

Josephus Divjanovich

Anna Passavacz (!)

Georgius Bartholich

Josephus Shubich

Antonius Bartolich

Helena Jambreshich

Anna Sharich

Johannes Mariancsevich

Lucas Blaxanovich

Antonius Novakovich

Marianus Schneperger

Georgius Stipanovich

Colleta Paullich

Catharina Kattich

Helena Blagoevich

Maria Vukshich

Heva Csulich

Johannes Jurkovich

Martinus Miholich
Lucia Shepcsich

Adamus Pavunich

Johannes Littarich

Anna Pavoshevich

Catharina Pavishevich

Anna Glassovich

Margaretha Mrakovich

Colleta Zalovich

Francisca Kulauzovich

Bonus Mikolashevich

Johannes Biuklich

Hieronymus Biuklich

Martinus Biuklich

Johannes Xivkovich

Franciscus Knexevich

Adamus Kessarich

Antonius Vinkovich

Josephus Begovich

Catharina Orshich

Catharina Lovrich

Nicolaus Dilacs

Josephus Dilacs

Lucas Jakovcsevich

Michael Jakovcsevich

Stanislaus Kapetanovich

Josephus Kapetanovich

Antonius Kapetanovich

Stephanus Berkanich

David Spear

Antonius Novakovich

Rosa Lucich

Anna Mariancsevich

Magdalena Balatovich

Stephanus Lovrich
40

763

/p. 10/

Marcus Stipanovich

Helena Humilianovich

Heva Mathishich

Helena Zvanacz
Thomas Zecsevich

Lucas Petrich

Gregorius Petrich

Maria Gorik
42

Helena Balatovich

Catharina Kovacsevich

Johannes Benovich

Michael Benovich 
Heva Kovacsevich Helena Zidarovich Helena Blaxevich Helena Klobucsar Antonius Lukacsich Georgius Ivanishich Rosa Kovacsich Catharina Balatovich Anna Patajacz Catharina Kolarovich Johanna Vukomanich Catharina Kruljacz Brigita Prispilovich Thomas Gjurenovich Johannes Jakobovich Jacobus Benich Georgius Novoselacz Fabianus Jovich Stephanus Jovich Blasius Vidakovich Marcus Bennich Nicolaus Kvaternik Michael Tomashevich Philippus Novakovich Antonius Bartholovich Elias Lucsevich Mathias Lucsevich Marcus Lokovich Stanislaus Patajacz Johannes Pavishich Franciscus Vrabacz Lucas Marinich Johannes Schneperger Antonius Jurich Adamus Benakovich Clara Sharich Magdalena Gjuricsich Rosa Knexevich Johannes Ruscianacz Paullus Paljushich Michael Zecsevich
Anna Kvaternik

Maria Stefancsich

Maria Shimanovich

Barbara Verboshich

Johanna Sarcsevich

Magdalena Stojakovich

Magdalena Bartolovich

Helena Petrushich

Antonius Bosnjak

Marcus Balatovich

Blasius Benovich

Paullus Benovich

Marcus Matokovich

Elisabetha Perkovich

Agnes Gjanich

Martha Mastanievich

Josephus Biuklich

Nicolaus Jarshetich

Antonius Glersich (?)

Agnes Zvonarevich

Anna Zvonarevich

Anna Shoshich

Maria Kurtakovich

Heva Mihocsevich

Heva Matokovich

Maria Kovacsevich

Agnes Perokovich

Lucas Matakovich

Joseph Percsinovich

Michael Percsinovich

Catharina Lovoshevich

Magdalena Horvatovich

Maria Shteposhovich

Simon Matakovich

Petrus Gjumlovich

Emericus Benovich

Marcus Shimich

Martinus Gjumlovich

Vincentius Novoselacz

Catharina Abacsich
Thaddaeus Balatovich

Marianus Pritisanacz

Michael Herushich

Rosa Kovacsevich

Philippus Kapetanovich

Johannes Vinkovich

Georigus Mikcsich

Michael Ghergurich

Michael Jankirovich

Marcus Zidarovich

Paullus Zidarovich

Clara Littarich

Maria Littarich

Anna Glassovacz

Johanna Vidacsich

Rosa Vidacsich

Coleta Vratarich

Anna Mastanievich

Johanna Mastanievich

Stanislaus Mastanievich

Michael Benovich

Johannes Benovich

Marcus Gavran

Thaddaeus Stankov

Anna Joshavacz

Francisca Joshavacz

Johannes Verbushich

Paullus Cerncsanin

Georgius Bortich

Johannes Bortich

Laurentius Bortich

Stephanus Glavashevich

Johannes Blaxevich

Andreas Gjanich

Josephus Millinkovich

Michael Littarich

Lucas Shubich

Josephus Shubich

Petrus Shubich

Martha Mihich

Catharina Stefelich

45 
/p. 11/

Antonius Striskovich

Stephanus Gjuromedich

Helena Gjurcsich

Anna Gjurcsich

Catharina Kerpan

Helena Muhich

Ludmilla Hellmar

Antonius Vukoshich

Georgius Marincsich

Michael Barshich

Casparus Mareovich

Heva Cellich

Anna Kovacsevich

Anna Martinovich

Heva Cernovich

Magdalena Horvatovich

Lucia Jaznanacz (!)

Rosa Barshich

Magdalena Marich

Rosa Jovich

Michael Shimich

Anna Sarcsenich

Heva Majurich

Anna Ivanich

Maria Abranovich

Maria Humilakovich

Magdalena Humilakovich

Anna Prispilovich

Antonius Kolarich

Mathias Sersich

Hieronymus Jakobovich

Franciscus Mariancsevich

Franciscus Milinkovich

Marianus Ghergurich

Thaddaeus Dorich

Adamus Dorich

Jacobus Dorich

Antonius Vucsinovich

Antonius Vunovich

Theresia Kolarovich
Chatina Lovossevich

Magdalena Vidich

Petrus Barshich

Marcus Gjukanovich

Josephus Gjukanovich

Georgius Kossovich

Antonius Mihaljevich

Sibilla Vidich

Paullus Dashovacz

Andreas Dashovacz

Josephus Jazvanacz

Paullus Littarich

Antonius Mikenovich

Antonius Golich

Stephanus Matokovich

Antonius Poë

Antonius Shtimacz

Josephus Dabramovich

Antonius Kampich

Heva Stojcsich

Anna Stifanovich

Michael Noganovich

Johannes Noganovich

Anna Sarcsevich

Rosa Papula

Martinus Puhanich

Michael Puhanich

Stephanus Puhanich

Jacobus Shtimacz

Andreas Obranovich

Georgius Obranovich

Johannes Obranovich

Lucas Osvald

Marianus Kovacsich

Johannes Gjurkotich

Lucia Novakovich

Magdalena Novakovich

Anna Novakovich

Catharina Novakovich

Marcus Forintich
Antonius Csudanich

Antonius Gjuricsich

Magdalena Petanovich

Catharina Petanovich

Marcus Blaxanovich

Nicolaus Matoshich

Jacobus Kovacsevich

Michael Lukacsich

Josephus Lukacsich

Brigita Mihaljevich

Matthaeus Prispilovich

Jacobus Matakovich

Heva Stucsich

Maria Matoshevich

Anastasia Matoshevich

Juliana Barich

Juliana Gambathi

Francisca Boljkovacz

Anna Vicich

Marcus Matishich

Andreas Kruljacz

Fabianus Shimich

Matthias Shimich

Magdalena Barich

Barbara Kovacsich

Barbara Mikshich

Anna Rossandich (!)

Helena Rosandich

Magdalena Novakovich

Anna Gjanich

Magdalena Tomashevich

Catharina Novakovich ${ }^{23}$

Antonius Noganovich

Matthaeus Radinovich

Anna Matishich

Barbara Ikovich

Magdalena Shtimcsevich

Helena Schepcsich

Maria Spear

Maria Ghergurich

${ }^{23}$ Catharina Novakovich cancell. 


\begin{abstract}
Agnes Umilianovich Johannes Shaprak

Barbara Glasovacz
\end{abstract}

43

Catharina Starovich
Coleta Blagajacz
Hieronymus Matrakovich
Rosa Kovacsich
Marcus Ikovich
Francisca Ferkovich
Martinus Mihaljinovich
Matthaeus Knezovich
Maria Karapetrich
Georgius Basarich
Matthaeus Starcsevich
Franciscus Stefancsich
Andreas Gjanich
Johannes Rosandich
Simon Matokovich

Thaddaeus Millich

Martinus Kovacsevich

Maria Xivcsich

Michael Vinkovich

Maria Ikovich

Stanislaus Mikenovich

Matthaeus Markulich

Josephus Peicsevich

Magdelena Matessich

Lucia Xivesich $^{24}$

Georgius Puhanich

Maria Csekalovich

Matthaeus Gazicza

Matthaeus Stipanovich

Magdalena Vukoshich

Johannes Petanovich

Helena Jeho

Heva Blaxevich

Angelia Roxacz

Thomas Stuparich
Johannes Forintich

Petrus Shimanovich

Johannes Shimanovich

43

127

/p. 12/

Catharina Biljan

Maria Ghergurich

Johannes Mihaljevich

Angela Kovacsich

Barbara Petanovich

Michael Matovich

Petrus Milinkovich

Maria Pavlovich

Michael Spetich

Antonius Blaxevich

Georgius Ikovich

Johannes Marich

Lucas Umilianovich

Johannes Petanovich

Josephus Matokovich

Marcus Kovacsevich

Maria Abranovich

Gregorius Dobravacz

Marianus Matishkovich

Paullus Matokovich

Andreas Matokovich

Josephus Katavich

Franciscus Orozkovich

Barbara Oriovich

Maria Gorshich

Dorothea Ikovich

Johannes Lovoshevich

Jacobus Rosandich

Josephus Gherghich

Catharina Vidich

Lucas Petanovich

Coleta Gjukanovich

Rosa Majurich

Rosa Pletisanacz

Adamus Stuparich
Catharina Mihaljevich

Matthaeus Horvatovich

42

Lucia Blagajacz

Maria Matishich

Franciscus Mihaljevich

Birgita Vrabacz

Rosa Stanishich

Michael Mihaljinovich

Matthaeus Boxishkovich

Maria Berdarich

Marcus Rosandich

Matthaeus Radocsaj

Matthaeus Stefancsich

Josephus Vucsinovich

Petrus Rosandich

Johannes Shimich

Matthias Vuksinovich

Laurentius Jakovich

Catharina Gjurkotich

Johannes Blagajacz

Juliana Xivkovich

Matthias Mikenovich

Nicolaus Mihaljevich

Antonius Orozkovich

Maria Mathessich

Magdelena Mogus

Maria Horvatovich

Barbara Gorshich

Josephus Gazicza

Philippus Rosandich

Thaddaeus Knexevich

Barbara Kruljacz

Stanislaus Shtimacz

Josephus Sekulich

Heva Novakovich

Theresia Gjuretich

Lucas Lucsich

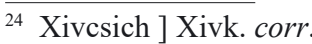


Michael Lucsich
Antonius Klaich
Petrus Shubich
Anna Peiscevich

39

Matthaeus Matoshevich
Magdelena Pintarich
Georgius Matkovich
Johanna Micsich
Francisca Marevich
Josephus Millinovich
Georgia Mikinovich
Johannes Stepanovich
Franciscus Thomashanac
Maria Mathianacz
Maria Koprivcsich
Maria Mikczova
Michael Marevich
Elias Vidakovich

Thaddaeus Prispilovich

Johannes Posavac

Nicolaus Jurkovich

Francisca Churcsich

Thaddaeus Zvekan

Marcus Stipanovich

Lucia Dorich

Catharina Stencsanin

Catharina Filipovich

Lucia Filipovich

Michael Filipovich

Matthaeus Mihaljevich

Anna Petrovich

Matthias Garich

Michael Gjuranich

Josephus Ivancsich

Helena Ozmarla

Georgius Gherbushich

Josephus Stefancsich

Matthias Stefancsich

Franciscus Stefancsich

Nicolaus Xivcsich

Matthias Remetich
Johannes Martinovich
Marcus Sharich
Lucia Benovich
Johannes Matoshevich

39

117

/p. 13/

Marcus Ghergoshevich

Rosa Gjunek

Margaretha Sartich

Johanna Lovossich

Johannes Biljan

Georgius Matokovich

Anastasia Sudarich

Antonius Thomashanacz

Franciscus Ognjanovich

Lucia Kovacsevich

Anastasia Ivanovich

Franciscus Thomashanac

Antonius Blaxevich

Magdalena Stankovich

Josephus Prispilovich

Stephanus Kovacsevich

Matthias Jerkovich

Maria Filipovich

Fabianus Stojicsich

Catharina Pritishanacz

Theresia Dorich

Matthias Mihaljovich

Heva Filipovich

Antonius Cernovich

Franciscus Pritishanacz

Rosa Bartolovich

Matthias Ivancsich

Thomas Benich

Adamus Gjuranich

Dorothea Ghergurich

Helena Quaternik

Barbara Vremich

Georgius Stefancsich

Georgius Boxishkovich

Michael Shimich

Petrus Blagoevich

Elias Matishich
Marianus Josipich

Marcus Kaprianovich

Elisabetha Pavoshevich

Nicolaus Matoshevich

39

Magdalena Kolarich

Barbara Mihashich

Johanna Stipanovich

Francisca Grubesh

Petrus Biljan

Michael Matokovich

Josephus Sundalich

Nicolaus Thomashanac

Francisca Bertich

Anast[as]ia Antunicseva

Catharina Mihaljevich

Johannes Petrovich

Johannes Mikó

Catharina Stankovich

Antonius Posavacz

Matthaeus Jurkovich

Rosa Ciprianovich

Magdalena Gumlich

Michael Osselich

Maria Dorich

Maria Garich

Matthias Vidakovich

Maria Turanovich

Michael Iliich (!)

Heva Glasovac

Johanna Xivkovich

Paullus Kacsich

Andreas Marshich

Matthias Petrovich

Maria Abramovich

Simon Kattich

Michael Stefancsich

Andreas Haracsich

Nicolaus Shimich

Josephus Matheshich

Martinus Gjuricsich

Georgius Pavich 


\author{
Marianus Pavich \\ Thomas Thomashevich \\ Stephanus Sharich \\ Simon Shubich \\ Petrus Kovacsevich
}

42

Magdalena Mastanievich
Clara Mastanievich
Rosa Abranovich
Barbara Rebusha
Thomas Matishich
Catharina Bellan
Georgius Verboshich
Johanna Maljevich
Johannes Vukshich
Barbara Cernik
Josephus Novakovich
Franciscus Gjuretich
Francisca Majurich
Matthias Dorich
Johannes Paskalich
Johannes Ceppak
Johannes Blash
Heva Benovich
Catharina Franich
Lucas Stampor
Heva Millinkovich
Jacobus Babich
Marcus Majurich
Josephus Novakovich
Lucas Vrabac
Petrus Tomenovich
Franciscus Shubich
Catharina Gorshich
Maria Loncsarevich
Michael Brodar
Helena Ghergurich
Helena Johovich
Angela Matokovich
Anna Mullich
Franciscus Petanovich
Matthias Abicsich

Johannes Zecsevich

Marianus Thomashevich

Marianus Remetich

Georgius Biuklich

Georgius Prispilovich

42

126

/p. 14/

Maria Mastanievich

Helena Shtimac

Anna Rebusha

Paullus Littarich

Catharina Rebusha

Maria Kurtakovich

Theresia Maljevich

Antonius Stojakovich

Fabianus Vukshich

Abrahamus Kasapovich

Adamus Ivanovich

Magdalena Shtimacz

Maria Divianovich

Maria Shorgavich

Michael Perokovich

Franciscus Dorich

Stephanus Groxich

Catharina Stephanovich

Lucia Stojich

Lucas Mucsich

Juliana Mihaljovich

Paullus Makxich

Martinus Novakovich

Simon Novakovich

Stephanus Lockmir

Marcus Shtimac

Marcus Czvitkovich

Maria Jurkotich

Matthias Brodar

Thomas Lucsich

Helena Shorga

Rosa Biuklich

Anna Brodar

Magdalena Lovoshevich

Josephus Bodak

Paullus Millinkovich
Matthias Thomashevich

Josephus Klarich

Andreas Shubich

Johannes Borissa

Magdalena Shtimacz

42

Coecilia Mastanievich

Anna Ergovich

Catharina Rebusha

Paullus Sidar

Dorothea Muvich

Antonius Xivcsich

Jacobus Stojakovich

Magdalena Shokicsich

Maria Mikicsevich

Nicolaus Kasapovich

Franciscus Mihich

Rosa Vukovich

Elias Dorich

Michael Boghetich

Maria Balatovich

Maria Volarovich

Michael Lastavich

Johanna Blaxicsevich

Oliva Benovich

Georgius Stampor

Josephus Starcsevich

Georgius Ghergurich

Franciscus Novakovich

Johannes Vrabac

Jacobus Biuklich

Thomas Mihaljevich

Johannes Klaich

Magdalena Jurkotich

Johannes Brodar

Georgius Noganovich

Maria Kvatacsek

Helena Matokovich

Magdalena Gavranich

Catharina Malovich

Antonius Stancsich

Matthias Lovrich 


\begin{abstract}
Matthias Sigur
Marcus Mastanievich

Anna Benovich

Johannes Perokovich

Francisca Bernich
\end{abstract}

41

Antonius Maljevac
Johannes Littarich
Marcus Billan
Marcus Mihaljevich
Georgius Lastrovich
Johannes Shtimac
Magdalena Kapraljevich
Maria Kovacsevich
Franciscus Banjakovich
Thomas Nikolashevich
Maria Mihich

Anna Kovacsevich

Francisca Stojakovich

Maria Kovacsevich

Rosa Marcsich

Michael Iliich (!)

Johannes Shtroncz

Antonius Zdelarovich

Simon Magleshich

Antonius Filipovich

Marcus Johovich

Maria Boshich

Catharina Tüppelhoffer

Dorothea Udovicsich

Johannes Jakshich

Petrus Ciprianovich

Bartholomaeus Pejcsevich

Heva Mishich

Johannes Sharcsich

Matthias Stojicsich

Magdalena Stetich

Georgius Sandorovich

Josephus Biuklich

Magdalena Franich

Thomas Kovacsich

Catharina Jelich
Athanasia Matishich

Simon Mastanovich

Catharina Knexevich

Martinus Perokovich

Rosa Blaxich

41

123

/p. 15/

Bartholomaeus Maljevac

Marianus Begovich

Martinus Mihaljevich

Martinus Shibalia

Johannes Mogush

Michael Shnaperger

Johanna Knexevich

Johanna Mihich

Matthias Glavashevich

Blasius Gjanich

Catharina Skvoshevich

Anna Kampich

Catharina Mishich

Magdalena Koprivesevich

Rosalia Majerin

Dominicus Cvitovich

Heva Pecsinovich

Paullus Mateshich

Maria Kozevich

Michael Jazvanac

Michael Bortich

Rosa Horvatovich

Catharina Hertmann

Stephanus Becsky

Matthias Glassovac

Johannes Mayer

David Mishich

Josephus Stankovich

Johannes Biljanac

Magdalena Milloshevich

Georgius Sandorovich

Anna Golubovich

Paullus Becharevich

Cecilia Benich

Andreas Bajukovich

Maria Lovoshevich
Maria Shimkovich

Josephus Mastanovich

Jacobus Mastanovich

Johannes Shtimacz

Antonius Sarshich

41

Magdalena Maljevac

Johannes Milcsich

Nicolaus Barilovich

Paullus Lastovich

Magdalena Kerpan

Adamus Shneperger

Georgia Horvatovich

Johannes Banjakovich

Andreas Puhanich

Michael Majurcsevich

Anna Abacsovich

Catharina Garagich

Catharina Kovacsevich

Anna Paullovich

Johannes Kottner

Petrus Koppis

Barbara Knopf

Michael Magleshich

Heva Kapetanovich

Georgius Gorshich

Martinus Martincsich

Johannes Marelich

Coleta Blaxevich

Martinus Jaznovich

Jacobus Ciprianovich

Adamus Knexevich

Johanna Mihaljevich

Marcus Shikanich

Petrus Littarich

Franciscus Peich

Petrus Franaetovich

Maria Shikanich

Antonius Mariancsevich

Magdalena Russich

Catharina Matanich

Josephus Ocellich 


Matthias Bosnjakovich
Agatha Glasovich
Georgius Mihich
Antonius Pritzel

40

Blasius Mathoshich
Elisabetha Mihallinger
Elisabetha Tantzós
Catharina Redlin
Theresia Shucsin
Margaretha Tucanich
Barbara Mateshich
Johannes Stankovich
Antonius Zidar
Petrus Ivanovich
Petrus Starcsevich
Jacobus Puhanich
Matthias Vukovich
Josephus Stipanovich
Nicolaus Johovich
Thomas Sharich
Antonius Stojicsich

\author{
Fabianus Filippovich \\ Catharina Zalovich \\ Michael Saracsich \\ Josephus Eisner
}

40

120

/p. 16/

Petrus Majstorovich

Anna Trankel

Magdalena Mayerinn

Margaretha Volarevich

Johannes Ocsevich

Barbara Gheghich

Oliva Stojicsich

Johannes Vertich

Georgius Ivanovich

Maria Kossovich

Adamus Marinkovich

Georgius Gjurich

Petrus Johovich

Franciscus Blaxevich

Marcus Mastanievich

Marcus Sharich

Thomas Stojicsich

Heva Sharich
1777.

18

52
Maria Bartholovich

Josephus Mihich

Antonius Pavoshovich

Johannes Gjukanovich

40

\author{
Thomas Boxich \\ Adamus Erich \\ Elisabetha Shokcsevich \\ Veronica Majstorovich \\ Martinus Divianovich \\ Anna Jogovich \\ Cecilia Peich \\ Johannes Knexevich \\ Blasius Matheshich \\ Johanna Gjuretich \\ Benedictus Markulich \\ Johannes Agrekovich \\ Georgius Lastavich \\ Mihael Johovich \\ Elias Benich \\ Johannes Stojicsich \\ Thomas Sharich
}

Summa omnium $1565^{25}$

Izvor: Arhiv Franjevačkog samostana sv. Antuna Padovanskog u Našicama, Matična knjiga vjenčanih župe Našice od 1717. do 1787.

\section{Kontakt autora:}

dr. sc. Milan Vrbanus, znanstveni savjetnik

Hrvatski institut za povijest - Podružnica za povijest Slavonije, Srijema i Baranje, Ante Starčevića 8, 35000 Slavonski Brod

e-mail: mvrbanus@isp.hr; mdirektor@net.hr

${ }^{25}$ Ukupni zbroj krizmanika u župi Sv. Antuna Padovanskog u Našicama nije točan. U našičkoj župi zagrebački biskup Josip Galjuf podijelio je sakrament potvrde za 1562 krizmanika, a ne 1565 , kako je navedeno u popisu. 
